\title{
Calibration Concept for Dimensional Measurements with Optical Multiscale Multi-Sensor Systems
}

\author{
A. Loderer, W. Hartmann, T. Hausotte \\ Institute of Manufacturing Metrology, Friedrich-Alexander Universität Erlangen-Nürnberg (FAU), \\ Nägelsbachstraße 25, 91052 Erlangen, Germany \\ andreas.loderer@fau.de
}

\begin{abstract}
:
This article describes a new calibration concept for dimensional measurements of optical measuring systems. Using the example of a prototypical multiscale multi-sensor fringe projection system for production-related inspections of sheet-bulk metal formed parts, current measuring procedures of the optical system are introduced. Out of the shown procedures' deficits a new concept is developed for determining the orientations and positions of the sensors' measuring ranges in a common coordinate system. Principle element of the concept is a flexible calibration artefact, adapted on the measuring task of the fringe projection system. By measuring the calibration artefact with each fringe projection sensor and aligning the resulting datasets on a digital reference model of the artefact, sensor specific transformation matrices can be calculated which allow transforming the sensors' datasets into a common coordinate system. Thus geometrical relation between individual measured features can be determined and dimensional measuring beyond the measuring range of a sensor became possible. Due to series of experiments the advantages of the new calibration concept in comparison with the current measuring procedures are finally revealed.
\end{abstract}

Key words: Fringe projection, multi-sensor, dimensional measurement, multiscale, calibration

\section{Introduction}

New production technologies, like sheet-bulk metal forming [1], involve new challenges for dimensional measurements of the manufactured parts. In the case of sheet-bulk metal forming metrological requirements of a production-related inspection arise from the short cycle time, the complex and filigree geometry and varying surface roughness due to the high, irregular distributed forming forces [2]. The challenges of inspecting complex workpieces can be explained by considering the "golden rule of measuring metrology" [3]. In 1968 Georg Berndt developed a rule for selecting appropriate measurement systems. Therefore, the measurement uncertainties of the measurement systems have to be known. Following the recommendation of the "golden rule", the measurement uncertainty should be at least less than a fifth, better less than a tenth, of the tolerance width. If this minimum requirement can be met, it is assured that the measurement results are accurate enough. To achieve these requirements, a prototype of a multiscale multi-sensor fringe projection system was developed, designed for a productionrelated environment (see Fig. 1.).

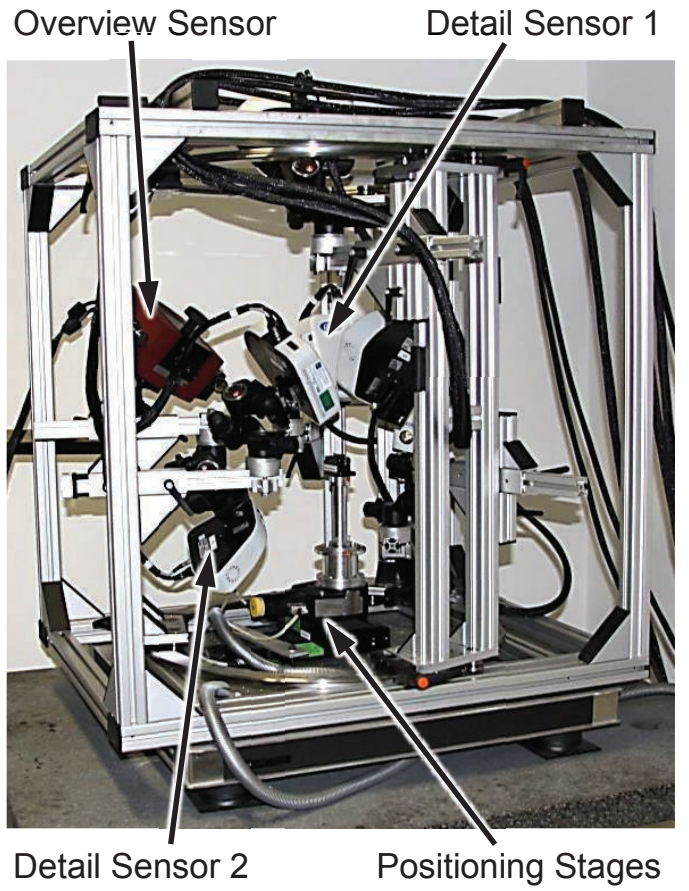

Fig. 1. Setup of the prototypical multiscale multisensor fringe projection system.

The main parts of the prototype systems are three different types of fringe projection sensors 
with varying measuring range and resolutions (see Tab. 1.).

To get an overview of the workpiece and also to measure large features at once, an exchangeable fringe projection sensor with a measuring range of the size of the workpiece is installed [4]. For the measurement of filigree elements, two other types of fringe projection sensors are used as detail sensors which can be arranged around the workpiece. Each sensor captures only one feature, but in a resolution, adapted to the feature's size [5].

Tab. 1: Technical specifications of the fringe projection sensors.

\begin{tabular}{|l|c|c|}
\hline $\begin{array}{l}\text { Sensor } \\
\text { type }\end{array}$ & $\begin{array}{c}\text { Measuring } \\
\text { range in } \mathrm{mm}\end{array}$ & Resolution in $\mu \mathrm{m}$ \\
\hline $\begin{array}{l}\text { Overview } \\
\text { Sensor }\end{array}$ & $115 \times 88 \times 92$ & $\begin{array}{c}21 \text { (mean point } \\
\text { spacing) }\end{array}$ \\
\hline Detail & $13 \times 10 \times 3$ & $\begin{array}{c}17 \text { (lateral) } \\
\text { (vertical) }\end{array}$ \\
Sensor 1 & & $\begin{array}{c}2.5 \text { (lateral) } \\
0.3 \text { (vertical) }\end{array}$ \\
\hline Detail & $4 \times 3 \times 1$ & \\
Sensor 2 & &
\end{tabular}

The process of gathering measuring results out of multiscale datasets can be divided into four main steps (see Fig. 2.): Firstly, measurements were done by all selected fringe projection sensors automatically. Then the sensors' datasets have to be transformed into a common coordinate system and merged together. Therefore the datasets are roughly aligned in a coarse registration by selecting corresponding points in each dataset manually. Only if there is a large overlapping area with at least one significant feature, an automatic algorithm, presented in [6], can be considered, but often this requirement cannot be met. In contrast to the following fine registration various automatic algorithms are available. The last step of the standard measuring procedure is the evaluation which can be also performed automatically. The presented procedure requires at least a small overlapping area. However, even if enough corresponding points are available, the procedure is neither fast nor accurate enough to benefit from the high accuracy of the fringe projection sensors.

In order to get a reliable geometrical relation between individual measured features and the ability to transform datasets without overlapping areas into a common coordinate system, a calibration concept, adapted on the properties of the prototypical multi-sensor multiscale measuring system, has to be developed.

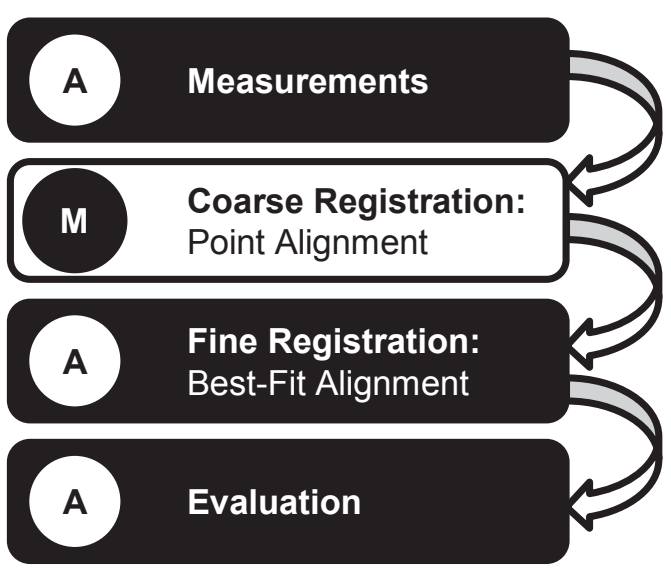

Fig. 2. Automatic (A) and manual (M) steps of the standard measuring procedure.

\section{Calibration Principle}

For proving the basic idea of the calibration of optical multiscale multi-sensor systems a flat calibration artefact was used (see. Fig. 3). On the calibration artefact's surface lines of differing distances as well as radii of differing sizes were milled in and thereby a unique surface structure could be created [7].

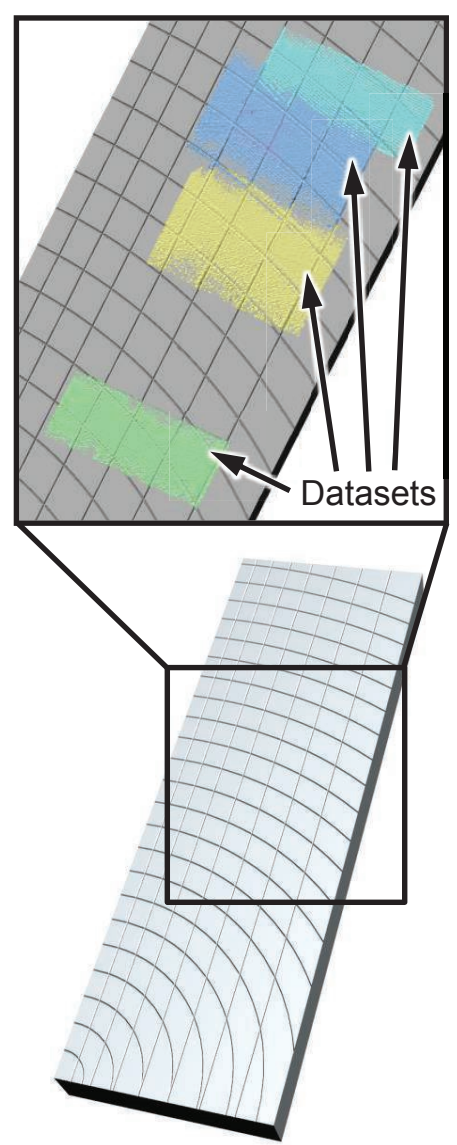

Fig. 3. Flat calibration artefact for testing the calibration principle.

Measuring ranges of the considered optical sensors are significantly smaller than the 
artefact's size. Setting up a multi-sensor measurement, the sensors' measuring ranges are positioned onto a measuring object. Subsequently, the measuring object is replaced by the calibration artefact. Each sensor measures now a part of the calibration artefact's surface and due to the unique surface structure, the position of each dataset can be allocated. By a manually coarse registration using point alignments and a following automatically fine registration, each dataset can be aligned on a CAD-model of the calibration artefact. All necessary transformations to get the datasets in the correct positions can be expressed in transformation matrices. These matrices represent the calibration of the sensors and have to be saved. Replacing the calibration artefact by a measuring object, again the datasets of each sensor can be transformed in the correct position by using the transformation matrices of the sensor. If the measuring range of fringe projection sensor is changed, the calibration procedure has to be done once more.

The important advantage of the calibration principle is the loss of need for corresponding areas. Even not overlapping datasets can be located correctly and thereby dimensional measurements with optical multiscale multisensor systems are enabled. Moreover, the time-consuming manual coarse registration has to be done only in the calibration procedure. Once all transformation matrices are available, the steps of the measuring procedure run automatically (see Fig. 4.).

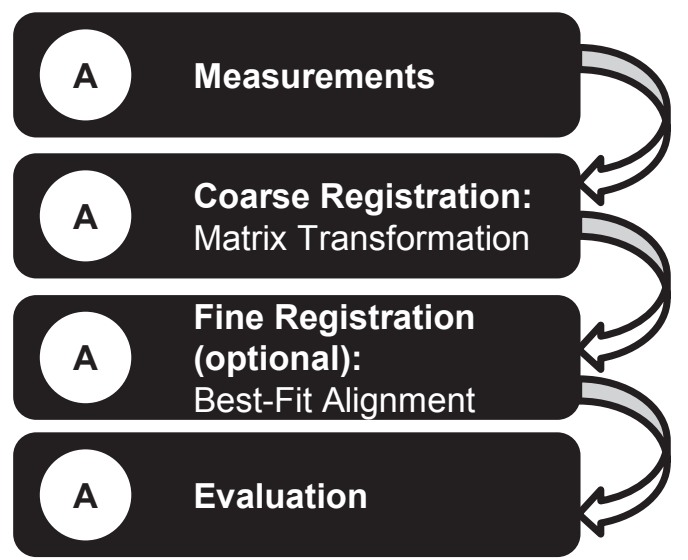

Fig. 4. Automated (A) measuring procedure by using transformation matrices.

\section{Flexible Calibration Concept}

A crucial disadvantage is the flat shape of the calibration artefact. Sheet-bulk metal formed objects, the prototypical multiscale multi-sensor fringe projection system is designed for measuring, often are of round shapes with varying diameters (see Fig. 5). With a flat calibration artefact, the fringe projection sensors can only be calibrated if their measuring ranges are positioned on the same height and orientated similar. However, complex features like cylinders require differently positioned sensors with differing heights of their measuring ranges. The flat calibration artefact is not capable for these demands.

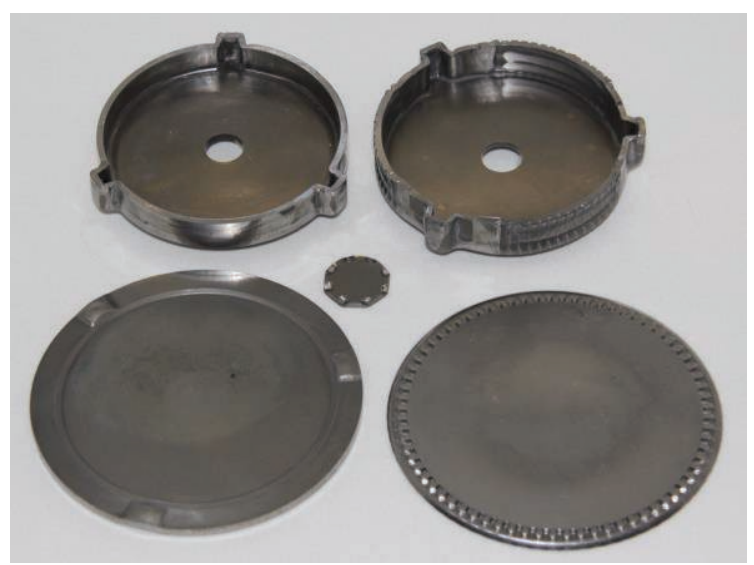

Fig. 5. Selection of sheet-bulk metal formed parts of differing sizes and shapes.

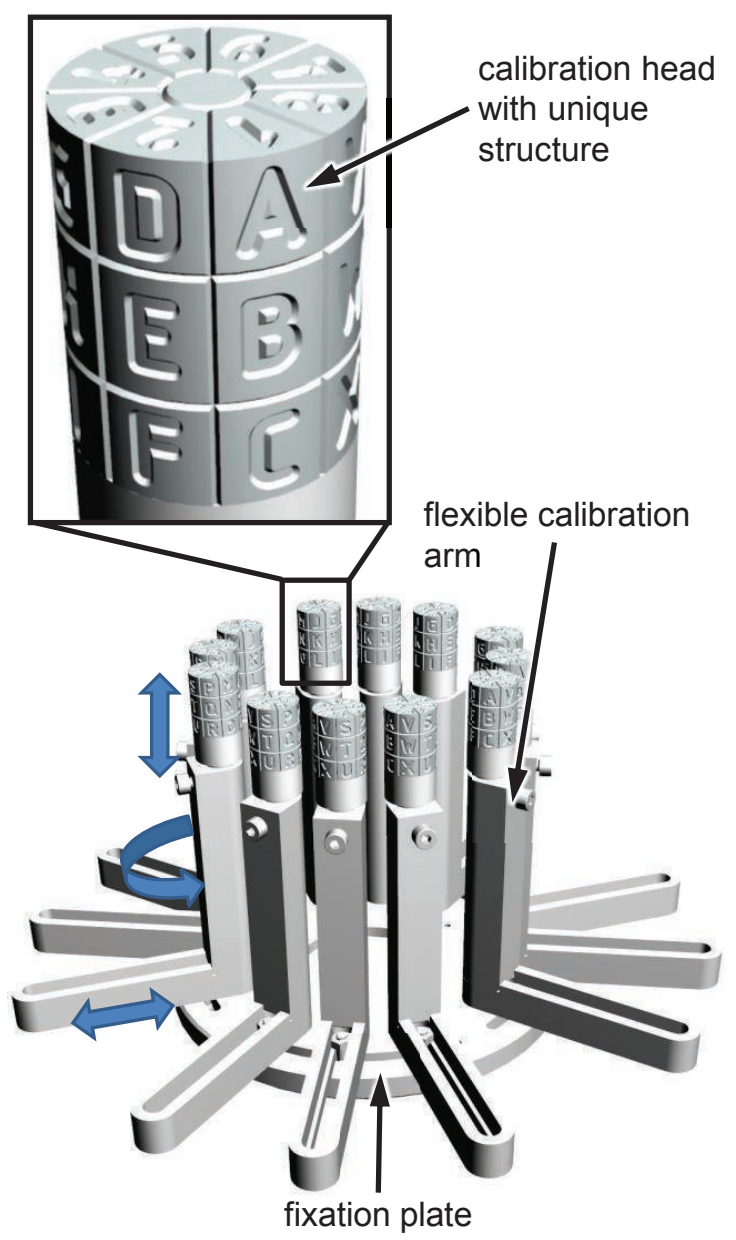

Fig. 6. Flexible calibration artefact adapted on sheet-bulk metal formed parts. 
Thus a flexible calibration concept was worked out to allow also dimensional measurement of complex features by using optical multi-sensor systems. This concept mainly based on a new flexible and, adapted on the demands of sheetbulk metal forming, calibration artefact (see Fig. 6). The basic principle of the calibration artefact, which is a unique surface structure as well, can be found on cylindrical "calibration heads". These heads are mounted on calibration arms which are adjustable in lateral and vertical direction. Thereby, measuring ranges do not have to be set up in the same height, but rather can be oriented freely. In order to optimize the calibration heads for optical measuring systems, the surfaces are glass blasted to generate dull and well measurable surface structures.

According to the calibration concept, ten main steps have to be considered setting up a complete multi-sensor measurement (see Fig. 7). Firstly the measuring ranges of the fringe projection sensors have to be positioned on the measuring object. Then the measuring object is replaced by the calibration artefact and the calibration heads are positioned into the measuring ranges. At least one calibration head has to be inside the measuring range of each sensor. In the next step measurements of the fringe projection sensors are triggered. For generating a reference polygonal model of the calibration artefact, it is digitalized by using an optical sensor with a bigger measuring range. In the shown experiments the overview sensor is used for digitalizing the calibration artefact. Next the datasets of the fringe projection sensors are aligned to the digital reference polygonal model of the calibration artefact. Out of these alignments transformation matrices for each dataset are calculated which express the orientation of each fringe projection sensor in a common coordinate system. The calibration procedure finishes with replacing the calibration artefact by the measuring object. With measurements of the measuring object the following measuring procedure starts. Using the transformation matrices, the datasets of the fringe projection sensors can be transformed into the common coordinate system and merged to one common dataset. By repeating the measuring procedure this calibration concept enables holistic dimensional measurements of complex features.

\section{Comparison}

To detect the advantages, provided by the developed calibration concept, a comparison between the standard measuring procedure and the automated measuring procedure with the new calibration concept is worked out.

Positioning of measuring ranges on measuring object

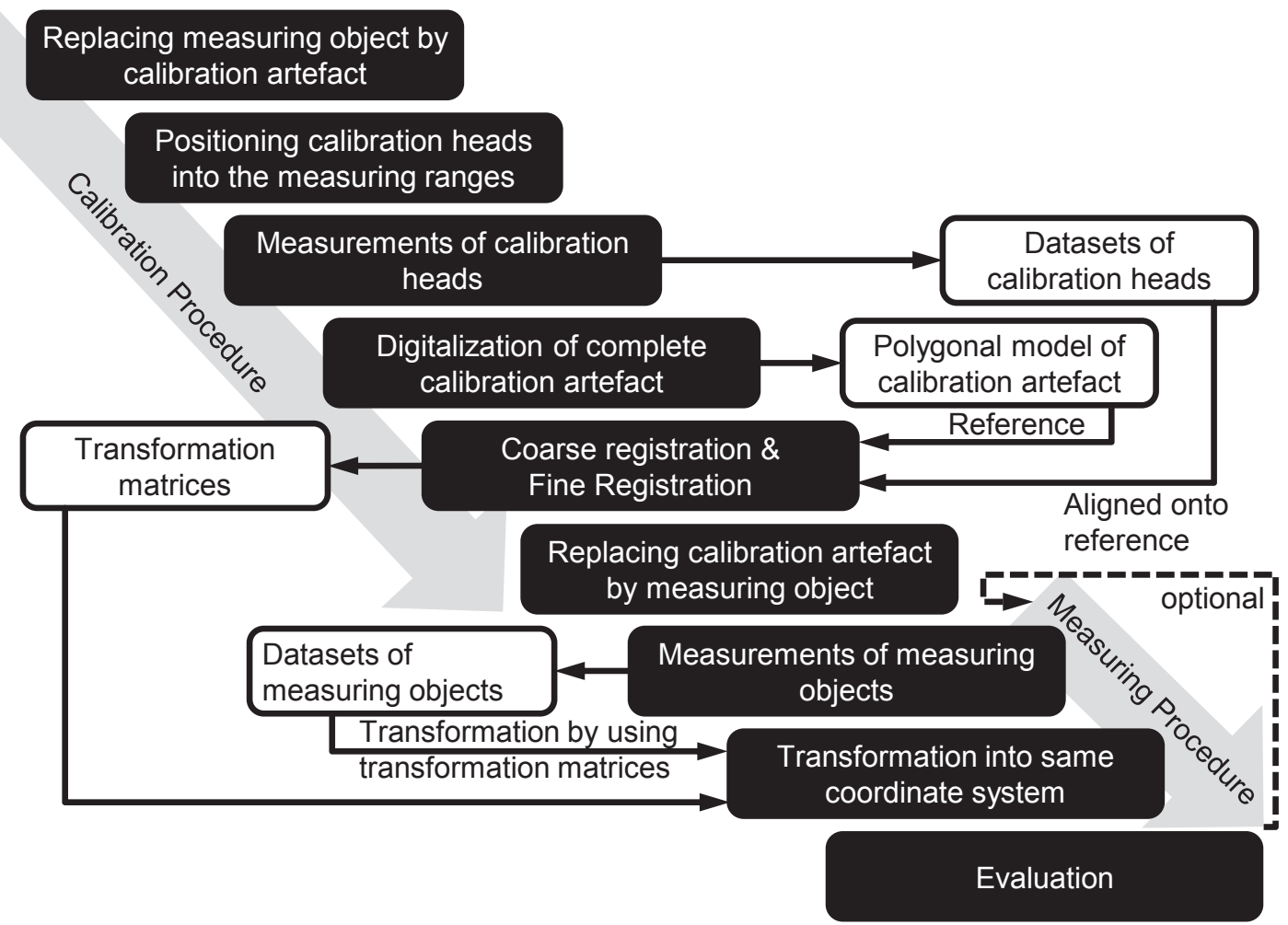

Fig. 7. Complete steps for calibration und measuring procedure. 
Therefore, the height and width of a step height standard have to be measured (see Fig 8). This measuring task represents the need of multisensor measurements: Due to optical effects like shadowing and technical limitations as e. $g$. the maximum thread angle, the measurement of both parameters by using only one fringe projection sensor is not possible. Only by changing the position of sensor or step height standard and performing more measurements, the features can be tediously detected by a single sensor. For a fast and reliable measurement more sensors with differing measuring positions are needed. In order to both measuring procedures, the deviations of height and width of the calibrated values are considered as parameters. The calculation of heights and width is done with Polyworks IMlnspect 2014. A consideration of DIN EN ISO 5436-1 for calculating step heights is not possible due to software restrictions. In addition to both, the time needed for performing all necessary steps is evaluated.

The results for the deviation of width show a significant difference between the automated measuring procedure with the according new calibration concept and former the standard
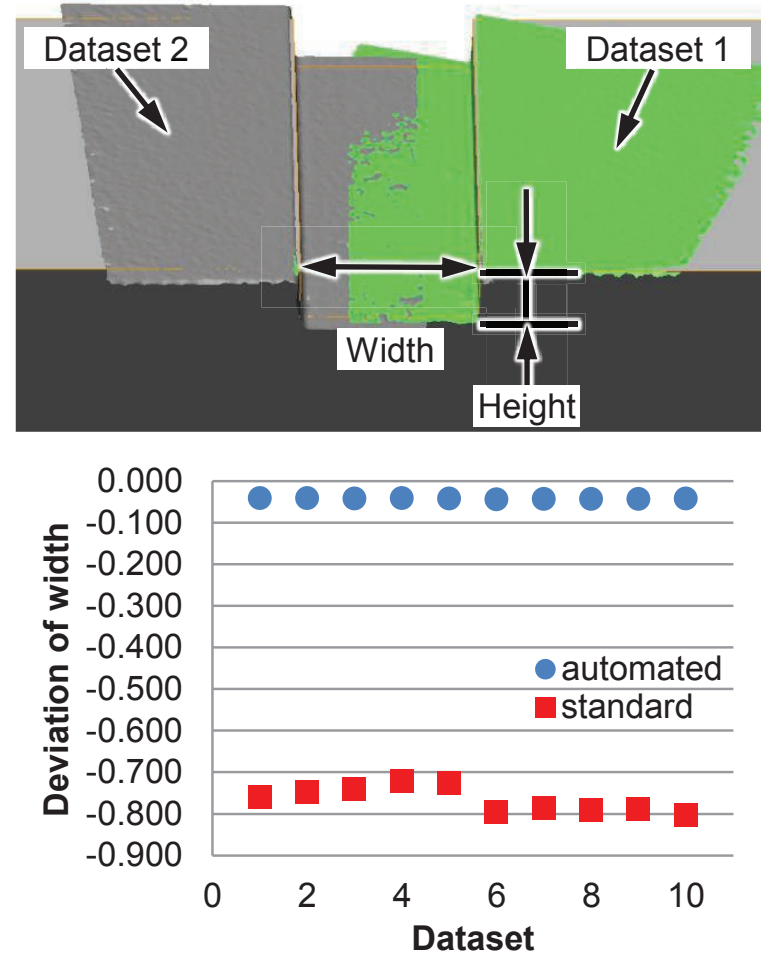

(1) Positioning and
measuring
calibration heads measuring procedure. Whereas the measuring results, gathered by using the standard measuring procedure, are between $0.7 \mathrm{~mm}$ and $0.8 \mathrm{~mm}$ smaller than the calibrated value, the deviation averages $-0.42 \mu \mathrm{m}$ using the automated measuring procedure. Considering the deviation of height, there is a difference between both procedures. The deviation averages $5 \mu \mathrm{m}$ for the automated and $-3 \mu \mathrm{m}$ for the standard procedure. Although this difference seems to be small, it's statistical significance is proved by using a student's t-test. However, focusing on the results' distributions, the reliability of the automated measuring procedure becomes obvious. The automated procedure provides continuously the same value for results, whereas the values of the standard procedure spread between $-5 \mu \mathrm{m}$ and $3 \mu \mathrm{m}$.

Comparing the duration needed for performing all required steps, the automated measuring procedure takes less time. Even though the detected difference is only about two minutes, the automated process is of advantage the more datasets are used.
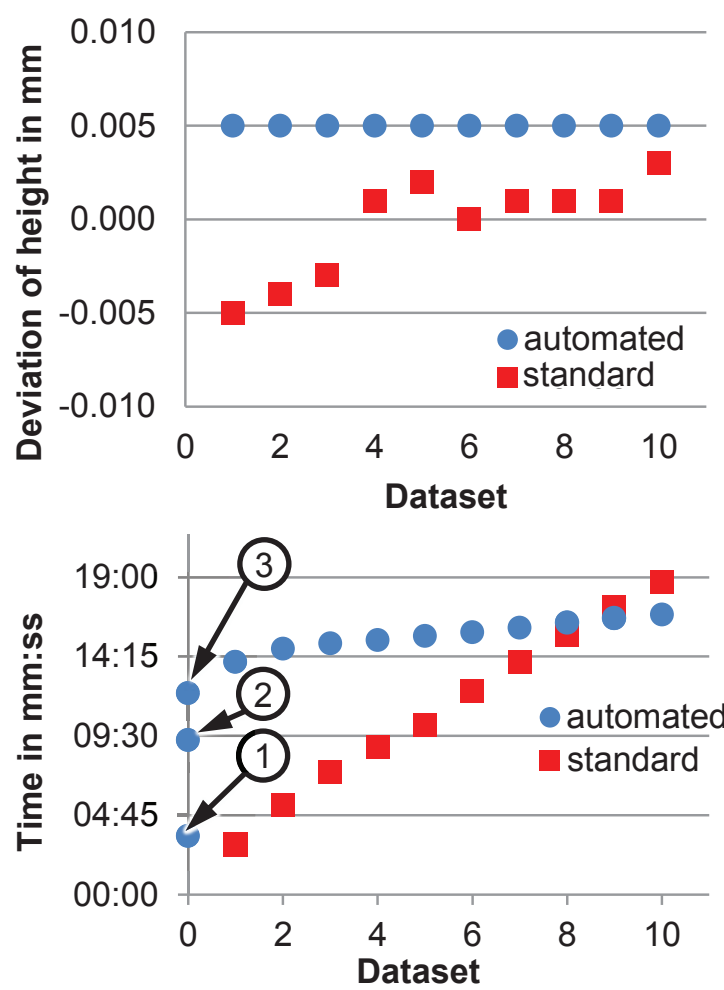

Fig. 8. Considered parameters for comparing standard measuring procedure (standard) and automated measuring procedure (automated) with new calibration concept (upper left corner). Results for deviations of height (upper right corner), width (lower left corner) and comparison of needed time (lower right corner). 
Whereas the needed time for the standard measuring process increases nearly linearly and one time step represents one competed dataset, the most time-consuming steps of the automated procedure are steps one to three. These steps belong to the calibration procedure and include the positioning and measuring of the calibration heads, the digitalization of the calibration artefact and the registration of the calibration heads' datasets in order to calculate the transformation matrices. Once the matrices are available and a script for an automated measuring process is created, which is done in the fourth step, the needed time for the following datasets is significantly shorter.

\section{Conclusion}

In this article a new calibration concept, optimized for optical multi-sensor measuring systems, was introduced which allows dimensional measurements of features, larger than the measuring range of the optical sensor. The basic principle was proved by a flat shaped calibration artefact using the example of a prototypical multiscale multi-sensor fringe projection system. A unique surface structure of the calibration artefact ensures that the measured datasets can be aligned on a polygonal reference model and thus transformation matrices for each fringe projection sensor can be calculated. These matrices contain the positions and orientations of each sensor, expressed in a common coordinate system. Thereby the correct transformation of all measurement datasets in a common coordinate system is enabled in order to generate a holistic dataset. The basic principle was transferred to a flexible calibration artefact, adapted on the shape of sheet-bulk metal formed parts, for whose inspection the prototypical multiscale multi-sensor measuring system was designed for. Together with the new calibration and measuring procedures, an automated and reliable measurement of complex workpieces is now possible. Comparing the new calibration concept with the former standard measuring procedure by setting up a series of experiments, the gathered advantages become obvious.

While the advantages of the new calibration concepts were proved, its performance and limitations are not known yet. Therefore, in further investigations the calibration concept has to be characterized in detail and influences like the optical measuring system for digitalization and generating a polygonal reference model of the calibration artefact has to be evaluated. Aim of these investigations is the identification of limitations in order to specify, for which dimensional measurement task the calibration concept can be used best.

\section{Acknowledgements}

The authors are grateful to the German Research Foundation (DFG) for supporting the investigations in the research project "Manufacturing of complex functional components with variants by using a new metal forming process - Sheet-Bulk metal forming" (SFB/TR 73, online: https://www.tr-73.de). In addition special thanks should be expressed to Mr. Michael Harant of the Friedrich-AlexanderUniversität Erlangen-Nürnberg for his contribution as well as to Mr. Thomas Schneider, Mr. Daniel Gröbel, Mr. Philipp Hildenbrand and Mr. Johannes Koch from the Institute of Manufacturing Technology of the FriedrichAlexander-Universität Erlangen-Nürnberg.

\section{References}

[1] M. Merklein, J. M. Allwood, B.-A. Behrens, A. Brosius, H. Hagenah, K. Kuzman, K. Mori, A. E. Tekkaya, A. Weckenmann, Bulk forming of sheet metal, Annals of the CIRP, 2 (2012) 61, 725-745.

[2] M. Schaper, Y. Lizunkova, M. Vucetic, T. Cahyono, H. Hetzner, S. Opel, T. Schneider, J. Koch, B. Plugge, Sheet-bulk Metal Forming a New Process for the Production of Sheet Metal Parts with Functional Components, Metallurgical and Mining Industry, 3 (2011) 7, 53-58.

[3] G. Berndt, E. Hultzsch, H. Weinhild, Funktionstoleranz und Meßunsicherheit, Wissenschaftliche Zeitschrift der Technischen Universität Dresden, 17 (1986), 465-471.

[4] C. Ohrt, W. Hartmann, M. Kästner, A. Weckenmann, T. Hausotte, E. Reithmeier, Holistic measurement in the sheet-bulk metal forming process with fringe projection, Key Engineering Materials, 504 (2012), 1005-1010.

[5] A. Loderer, B. Galovskyi, W. Hartmann, T. Hausotte, Qualifying Measuring Systems by using Six Sigma, Proceedings of the 11th Global Conference on Sustainable Manufacturing Innovative Solutions, (2013), 143-148.

[6] L. Shaw, S. Ettl, F. Mehari, A. Weckenmann, G. Häusler, Automatic registration method for multisensor datasets adopted for dimensional measurements on cutting tools, Measurement Science and Technology, 24 (2013), 8pp.

[7] M. Kästner, T. Hausotte, E. Reithmeier, A. Loderer, C. Ohrt, P. Sieczkarek, Fertigungsnahe Qualitätskontrolle von Werkzeug und Werkstück, Tagungsband zum 2. Erlanger Workshop Blechmassivumformung 2013, 101-118.

[8] C. Ohrt, M. Kästner, E. Reithmeier, A. Weckenmann, J. Weickmann, Optische Inspektion von Blechmassivumformteilen und -werkzeugen mit feinen Nebenformelementen, Technisches Messen, 79 (2012) 2, 95-102. 